\title{
IMAGING DIAGNOSIS-AORTIC THROMBOEMBOLISM ASSOCIATED WITH SPIROCERCOSIS IN A DOG
}

- $\quad$ Robert M. Kirberger ${ }^{1}$,

- $\quad$ Anthony Zambelli ${ }^{1}$

${ }^{1}$ Department of Companion Animal Clinical Studies, Faculty of Veterinary Science, University of Pretoria, Private Bag X04, Onderstepoort 0110, Republic of South Africa. Address correspondence and reprint requests to Robert M. Kirberger, at the above address. E-mail: robert.kirberger@up.ac.za

[Figures and tables at the bottom of the document]

\begin{abstract}
An 8-year-old neutered female Rhodesian Ridgeback developed acute, nonneurological right pelvic limb lameness. Femoral pulsation was poor, and oscillometric blood pressure measurements between the two pelvic limbs differed markedly. A caudal aortic right external iliac embolus was detected sonographically. Radiographically, there was a caudal esophageal mass and thoracic vertebral spondylitis typical of spirocercosis. Using CT-angiography, a caudal thoracic aortic aneurysm with a mural thrombus was detected. The dog recovered following heparin and aspirin therapy but signs recurred 7 months later. Subsequently, the patient improved on treatment and remains asymptomatic. This report illustrates the value of CT-angiography in detecting aortic thrombosis in dogs with spirocercosis.
\end{abstract}




\section{History and Physical Findings}

AN 8-YEAR-OLD $45 \mathrm{~kg}$ neutered female Rhodesian Ridgeback developed acute right pelvic limb lameness. On examination, the right femoral pulse was absent, the limb was cool to the touch, and a nail cut to the quick bled poorly. Patellar reflexes and extensor strength were decreased on the right pelvic limb. Blood pressure was measured across the dorsal metatarsal artery using an oscillometric blood pressure monitor. ${ }^{*}$ Five readings were taken from each limb and averaged. Diastolic and systolic pressure averaged 40 and $76 \mathrm{mmHg}$, respectively, in the right pelvic limb and 90 and $132 \mathrm{mmHg}$, respectively, in the left pelvic limb.

\section{Laboratory data}

Fecal flotation with a modified sugar solution ${ }^{1}$ was negative for Spirocerca eggs. There was a mild thrombocytopenia $\left(179 \times 10^{9} / 1\right.$, normal range $\left.200-500 \times 10^{9} / 1\right)$. Results from routine serum chemistry testing, urinalysis, and coagulation profile evaluation were normal.

\section{Imaging}

Sonographically, the caudal aorta contained a 3-cm-long, mildly echogenic mass, which extended $6 \mathrm{~cm}$ into the right external iliac artery. The mass also appeared to extend into the median sacral artery. Color flow Doppler confirmed the absence of flow in this region. There was no sonographic evidence of altered cardiac chamber size or function, valvular growths, or free or intramural masses. On thoracic radiographs a 4-cm-diameter soft tissue opacity was present in the region of the caudal esophagus (Fig. 1). The 11th thoracic vertebra was characterized by mild ventral spondylitis. The aortic outline appeared smooth. These changes were consistent with spirocercosis. ${ }^{2}$ A CT examination $\dagger$ of the region between $\mathrm{C} 7$ and $\mathrm{L} 1$ was performed with the patient in sternal recumbency and under general anesthesia. Pre- and postcontrast images were reviewed in soft tissue (WL 32, WW 349) and lung (WL-400, WW 1600) windows. Sagittal and dorsal 2-mm-thick reconstructions were made as required. 
The esophageal mass was clearly delineated as it arose from the left ventrolateral aspect of the esophagus just caudal to the heart, extending caudodorsally to terminate just cranial to the gastroesophageal junction. The mass was $10 \mathrm{~cm}$ long and $5 \mathrm{~cm}$ in diameter at its widest point. There was no evidence of mineralization of the mass and it had moderate vascularization (Fig. 2). Specks of dorsal aortic intimal mineralization were seen at the level of T12. The aorta at the level of T12-13 had a 4-cm-long mural filling defect on its right ventrolateral aspect. The mass involved about $35 \%$ of the circumferential luminal wall and protruded $10 \mathrm{~mm}$ into the lumen (Figs. 2 and 3). The cranial portion of this filling defect appeared to arise from a slightly widened aorta, indicative of an aneurysmal dilatation. These changes were compatible to an aortic thrombus, secondary to aorta intimal damage caused by migrating Spirocerca lupi larvae. There was no evidence of pulmonary metastasis.

Doramectin injections at $0.5 \mathrm{mg} / \mathrm{kg}$ subcutaneously every 2 weeks for 12 weeks were planned to treat the $S$. lupi infection. ${ }^{3,4}$ Owing to poor client compliance, the second injection was given 3.5 weeks after the first, after which compliance improved. In addition, aspirin ( $6 \mathrm{mg} / \mathrm{kg}$ orally per day for 2 weeks) and low molecular weight heparin (50 IU/kg subcutaneously twice daily for $72 \mathrm{~h}$ ) were used to prevent ongoing thrombogenesis.

The clinical signs related to the iliac thrombosis resolved after 4 days and the dog continued to improve for 7 months before developing a mild relapse. The dog was clinically healthy otherwise and alert but had bilateral weak femoral pulses. Sonographically, the aorto-iliac thrombus was more extensive extending $3 \mathrm{~cm}$ more cranially with no evidence of blood flow past the site of embolization. Radiographically, the esophageal mass had resolved. Treatment with doramectin and aspirin at the same dosages and intervals was repeated and the patient was reportedly normal within 2 weeks. The patient continued to do well 3 months later.

\section{Discussion}

Canine aortic thromboembolism is rare. Reported causes are cardiac disease, neoplasia, glomerulonephropathy, atherosclerosis associated with hypothyroidism and hyperadrenocorticism, septicemic states, vegetative endocarditis, and corticosteroid 
therapy. These various etiologies result in a hypercoagulable state. ${ }^{5,6}$ Thromboembolism is more common in the cat where it is usually associated with dilated or restrictive cardiomyopathy. ${ }^{7}$

S. lupi typically results in a caudal esophageal granuloma, which may undergo neoplastic transformation. Associated intrathoracic abnormalities include spondylitis, aortic aneurysm, and intimal mineralization. ${ }^{2}$ Aorto-iliac thromboembolism associated with spirocercosis has been diagnosed previously in two dogs at necropsy. One was a Cavalier King Charles Spaniel ${ }^{8}$ and the other a Boxer, ${ }^{9}$ both of which died following unsuccessful treatment.

Caudal aortic thromboembolism in cats is often associated with cardiomyopathy, and leads to acute hindquarter paresis with pain, rhabdomyolysis, signs of peripheral vascular occlusion, and more serious systemic illness. ${ }^{7}$ Although some of the latter signs arise from cardiomyopathy, the release of intracellular substances from ischemic muscles (such as calcium, serum inorganic phosphate, and potassium) contributes to the general clinical picture. ${ }^{7}$ The concomitant release of platelet-derived thromboxanes and other chemical factors, as well as collateral blood supply, determine the different courses of hindquarter ischemic disease when compared with dogs in which this syndrome often causes chronic intermittent pelvic limb paresis. ${ }^{7,10,11}$

To the best of our knowledge, this is only the third reported aorto-iliac embolism secondary to spirocercosis-induced aortic thrombus and the first to image the primary thrombus in the thoracic aorta. Previously, a cranial abdominal aortic thrombus was

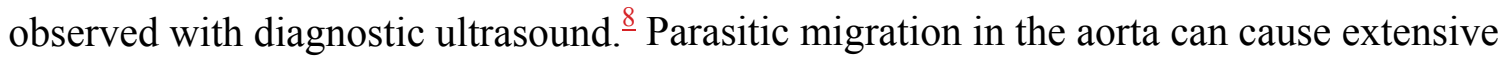
intimal damage and it is surprising that more instances of iliac thromboembolism are not encountered in endemic areas. There may well be a higher incidence of aortic thrombi but the lack of sophisticated imaging to date may be the reason why they have gone undiagnosed. Aortic aneurysm formation in the cranial abdominal aorta occurs rarely but abdominal ultrasound examinations in known spirocercosis patients should include evaluation of the abdominal aorta. CT is performed on S. lupi patients in our institution to determine possible malignancy as indicated by early mass mineralization ${ }^{2}$ or pulmonary metastasis and for surgical planning before mass removal. Based on the findings of this patient, imaging should include a CT-angiogram to evaluate subclinical or clinically 
evident aortic thromboembolism when there is suggestion thereof based on prior radiographic, clinical, or ultrasonographic findings. The aortic intima (in the thoracic portion) should be carefully examined for evidence of dilatation, stenosis, obstruction, or calcification. The diagnosis of early thrombotic disease may allow for better treatment with antithrombotic medications. On survey, thoracic radiographs' evidence of aortic aneurysm include aortic intimal calcification and an irregular outline of the aorta, particularly the descending aorta on VD or DV radiographs. ${ }^{2}$ The latter is commonly seen in patients in our hospital and these patients should be put on prophylactic antithrombotic therapy for at least 2 weeks. No evidence-based guidelines exist for canine patients suffering from thrombogenic diseases, but based on human experience with atherosclerosis of other causes, aspirin, heparins (particularly dexaparin), warfarin, or other medications may have a place. ${ }^{12,13}$ Given the length of the embolus, the blood flow velocity in the aorta, and the concurrent inflammation (thoracic intimal aortitis and within the thoracic granuloma), it is not surprising that the patient suffered such a dramatic clinical episode. Treatment of the primary pathogenic agent with doramectin is undoubtedly of some value in these patients.

Recanalization of the embolus may begin within hours of the initial event and may be more important in the normalization of regional blood flow than the development of new

collateral vessels. ${ }^{14,15}$ It is difficult to determine if in this patient the initial thrombus did not canalize over time or got canalized and then reoccluded.

Spirocercosis is an unusual cause of aortic thromboembolism and should be considered in dogs with acute or intermittent pelvic limb paresis in endemic areas. CT-angiography proved to be a useful diagnostic modality to determine the primary thrombus location as well as its size and other coexisting spirocercosis-associated pathology.

\section{Footnotes}

*Cardell Cardiometer, Sharn Veterinary Inc., Tampa, FL.

$\dagger$ Aquilon 16 slice helical CT, Toshiba Corporation Medical Systems Company, Tochigiken, Japan. 


\section{References}

1.

Markovics A, Medinski B. Improved diagnostics of low intensity Spirocerca lupi infection by the sugar flotation method. J Vet Diag Invest 2002;8:400-401.

2.

Dvir E, Kirberger RM, Malleczek D. Radiographic and computed tomographic changes and clinical presentation of spirocercosis in the dog. Vet Radiol Ultrasound 2001;42:119129.

3.

Lavy E, Aroch I, Bark H, et al. Evaluation of doramectin for the treatment of experimental canine spirocercosis. Vet Parasitol 2002;109:65-73.

4.

Berry WL. Spirocerca lupi oesophageal granulomas in 7 dogs: resolution after treatment with doramectin. J Vet Intern Med 2000;14:609-612.

5.

Dufort RM, Matros L. Acquired coagulopathies. In: Ettinger SJ, Feldman EC (eds):

Textbook of veterinary internal medicine (chapter 273). St. Louis: Elsevier Saunders, 2005;1929-1933.

6.

Fox PR, Petrie JP, Hohenhaus AE. Peripheral vascular disease. In: Ettinger SJ, Feldman EC (eds): Textbook of veterinary internal medicine (chapter 208).

St. Louis: Elsevier Saunders, 2005;1145-1165.

7.

Schoeman JP. Feline distal aortic thromboembolism: a review of 44 cases (1990-1998). J Feline Med Surg 1999;1:221-231.

8.

Gal A, Kleinbart S, Aizenberg Z, Baneth G. Aortic thromboembolism associated with Spirocerca lupi infection. Vet Parasitol 2005;130:331-335.

9.

Alvarenga J, Saliba AM. Iliac embolism in a dog. Mod Vet Pract 1971;52:37-38. 
10.

Brofman PJ, Thrall DE. Magnetic resonance imaging findings in a dog with caudal aortic thromboembolism and ischemic myopathy. Vet Radiol Ultrasound 2006;47:334-338. 11.

Boswood A, Lamb CR, White RN. Aortic and iliac thrombosis in six dogs. J Small Anim Pract 2000;41:109-114.

12.

Goodnight SH, Coull BM, McAnulty JH, Taylor LM. Antiplatelet therapy-Part II. West J Med 1993;158:506-514.

13.

Goodnight SH, Coull BM, McAnulty JH, Taylor LM. Antiplatelet therapy-Part I. West J Med 1993;158:385-392.

14.

Bick RL, Kaplan H. Syndromes of thrombosis and hypercoagulability. Congenital and acquired causes of thrombosis. Med Clin North Am 1998;82:409-415.

15.

Bick D, Fugger EF, Pool SH, Hazelrigg WB, Yadvish KN, Spence WC. Disseminated intravascular coagulation: pathophysiological mechanisms and manifestations. Semin Thromb Hemost 1998;24:3-18. 


\section{Figures and tables}

FIG. 1. Right lateral recumbent thoracic radiograph. Note the caudal esophageal mass (white arrows).

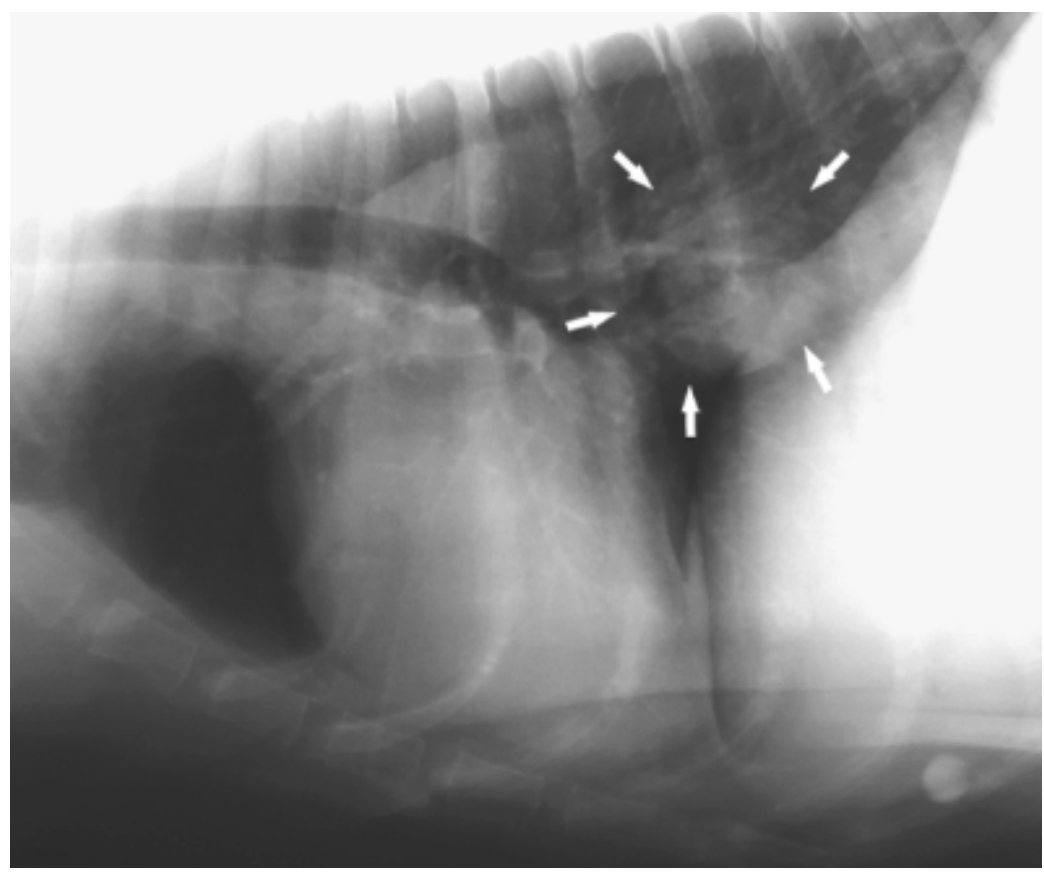


FIG. 2. CT-angiogram (WL 32, WW 349) sagittal reconstruction. Note the multilobulated irregular esophageal mass (white arrows) with conspicuous vessels, likely to be enlarged esophageal branches of the bronchoesophageal artery. There is mild aneurysmal dilation of the aorta at the origin of the ventrally located thrombus (black arrow heads).

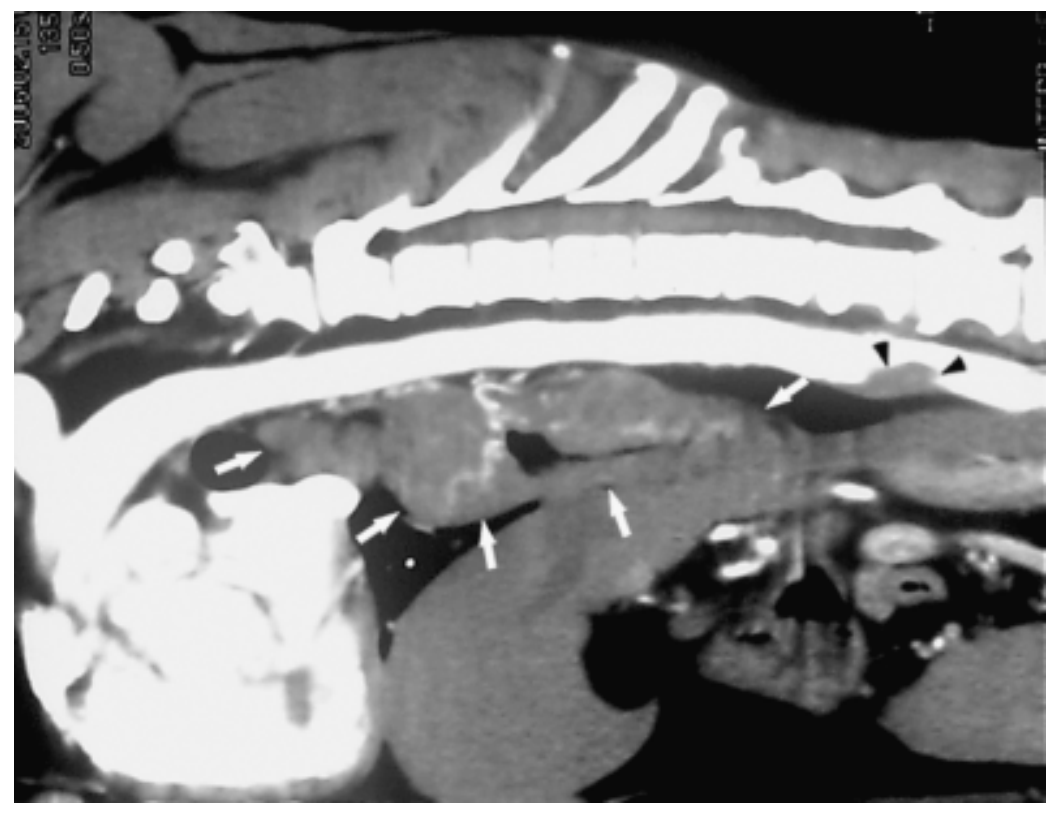

FIG. 3. Transverse CT-angiogram images of the aorta. (A) At level of caudal T12. (B) At level of caudal T13 illustrating aorta filling defect due to mural thrombus formation at 3 6 o' clock.
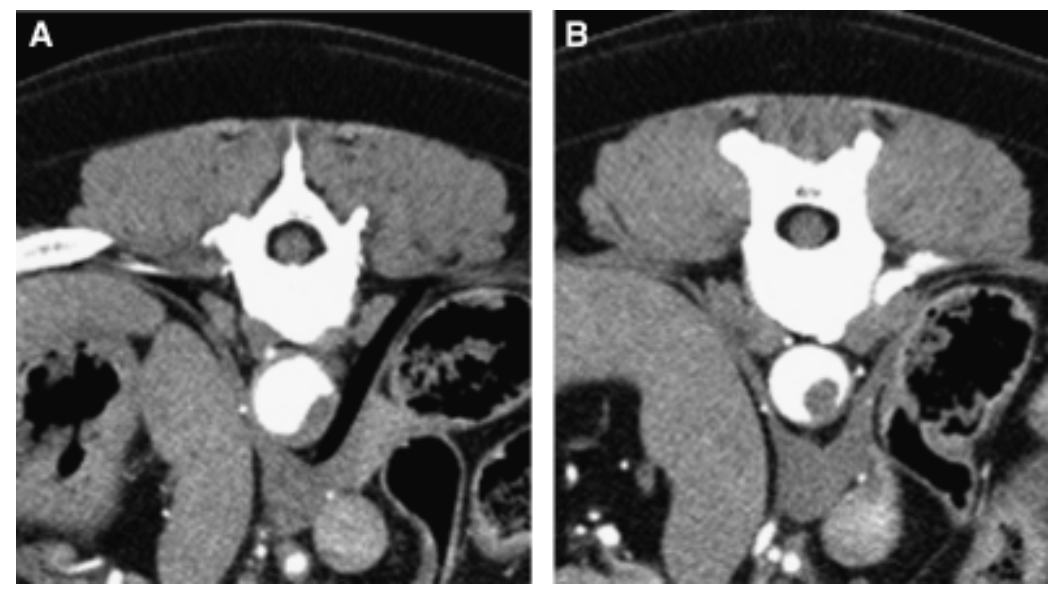